'Programa Fisiopatología, Instituto de Ciencias Biomédicas, Facultad de Medicina, Universidad de Chile, Santiago, Chile. ${ }^{2}$ Programa Virología, Instituto de Ciencias Biomédicas, Facultad de Medicina, Universidad de Chile, Santiago, Chile ${ }^{3}$ Departamento de Pediatría, Facultad de Medicina, Universidad de Chile, Santiago, Chile

${ }^{4}$ Centro de Salud Familiar Agustín Cruz Melo, Servicio de Salud Metropolitano Norte.

Departamento de Biología, Facultad de Ciencias, aMédico Veterinario, Universidad de Chile.

Trabajo financiado por Fondecyt proyecto 1090261

Recibido el 31 de mayo de 2012, aceptado el $21 \mathrm{de}$ diciembre de 2012

Correspondencia a: Dra. Patricia V. Díaz Programa Fisiopatología, Instituto de Ciencias Biomédicas, Facultad de Medicina Universidad de Chile.

Avda. Salvador 486 Teléfono: 56-2-2741560 Fax: 56-2-2741628 E-mail:pdiaz@med. uchile.cl

\section{Aumento de interleuquinas proinflamatorias y de cortisol plasmático en bronquiolitis por virus respiratorio sincicial: relación con la gravedad de la infección}

\author{
PATRICIA V. DÍAZ PhD ${ }^{1}$, ALDO A. GAGGERO ${ }^{2, a}$, RICARDO A. PINTO ${ }^{3}$, \\ ROSSANA MAMANI ${ }^{3}$, PAOLA A. UASAPUD ${ }^{4}$, MARÍA ROSA BONO $\mathrm{PhD}^{5}$ \\ Levels of inflammatory cytokines and \\ plasma cortisol in respiratory syncytial virus \\ bronchiolitis
}

Background: An increased inflammatory innate response may play a role in pathogenesis of respiratory syncytial virus (RSV) infection. Aim: To quantify proinflammatory cytokines (IL-6-IL-8, IL-1- $\beta$ and TNF- $\alpha$ ) in nasopharyngeal aspirate (NPA) and plasma, and plasma cortisol in previously healthy infants with RSV bronchiolitis. Patients and Methods: We studied 49 infants aged less than one year of age with RSV bronchiolitis and 25 healthy controls. Severity was defined using a previously described modified score. We quantified interleukins in NPA and plasma by flow cytometry and plasma cortisol by radioimmunoanalysis. Results: Among patients with RSV bronchiolitis, 25 were classified as severe and 24 as moderate or mild. Significantly higher levels of IL-6 and IL-8 in NPA and plasma and IL-1 $\beta$ in NPA were found in children classified as severe, when compared to those with moderate or mild disease and controls. There was a positive correlation between IL-6 and cortisol in plasma $(r=0,55 ; p<0,0001)$ and both were correlated with the severity of the disease. Conclusions: RSV bronchiolitis severity was associated with higher levels of inflammatory interleukins and plasma cortisol.

(Rev Med Chile 2013; 141: 574-581).

Key words: Bronchiolitis, viral; Interleukins; Respiratory syncytial virus infection.
$\mathrm{E}$ 1 virus respiratorio sincicial (VRS) es el patógeno que causa mayor morbilidad y mortalidad en lactantes menores ${ }^{1}$. Infecta anualmente a 3-7\% de adultos mayores sanos y entre 4-10\% de adultos con enfermedades concomitantes $^{2}$. La gravedad de la infección está relacionada con enfermedades pre-existentes tanto en niños ${ }^{3}$, como en adultos ${ }^{4}$. En lactantes previamente sanos sin factores de riesgo, la gravedad es muy variable y difícil de predecir. Los síntomas de la infección por VRS son variados desde manifestaciones leves de coriza, tos y fiebre hasta síntomas severos de obstrucción bronquial y bronconeumonía, con dificultad respiratoria, hiperinflación, sibilancias y signos de hipoxemia. La patogenia de la infección no se conoce totalmente ${ }^{5}$. Se sabe que el virus infecta las células epiteliales de la mucosa bronquial y se disemina de célula a célula a través de puentes intercitoplasmáticos formando sincicios o células multinucleadas. Como respuesta a la infección, las células epiteliales producen numerosas citoquinas proinflamatorias como interleuquina-8 (IL-8), interleuquina-1 (IL-1), interleuquina-6 (IL-6), factor de necrosis tumoral alfa (TNF- $\alpha$ ) y 
otros factores. Esta respuesta inflamatoria ha sido demostrada en lavados nasal y traqueal de niños infectados por $\mathrm{VRS}^{6-8}$, animales de investigación ${ }^{9}$, células de cultivo ${ }^{10}$, cultivos primarios de células de vía aérea ${ }^{11}$ y en células mononucleares de sangre periférica infectada in vitro por VRS ${ }^{12}$.

La causa de la gravedad de la infección aguda en lactantes previamente sanos, no está bien determinada. Algunos investigadores dan significado al desbalance Th-1/Th- $2^{13-16}$. En estudios anteriores, encontramos una disminución de la producción de IFN- $\gamma$ y de IL-12 por células mononucleadas en relación a la gravedad de la infección por VRS que se correlacionaba en forma indirecta con el aumento de cortisol plasmático ${ }^{17}$. Se ha postulado que la gravedad de la infección podría deberse a una depresión de la respuesta inmune específica celular ${ }^{18,19}$ y/o a un aumento de la respuesta inmune innata. Las interleuquinas pro inflamatorias IL-6, IL-8, IL-1 $\beta$ e TNF- $\alpha$ estimulan el eje hipotálamo-hipófisis-suprarrenal ${ }^{20-22}$. En voluntarios sanos se demostró que la infusión de IL-6 humana recombinante aumenta el cortisol plasmático ${ }^{23}$ sugiriendo que la IL-6 podría tener una acción antiinflamatoria.

Postulamos que en la infección por VRS se produce un aumento de cortisol plasmático que está relacionado con el aumento de interleuquinas pro inflamatorias y con la gravedad de la enfermedad. El objetivo de nuestro trabajo fue cuantificar en el aspirado nasofaríngeo y en el plasma de lactantes con infección respiratoria aguda baja (IRAB) la presencia de interleuquinas de respuesta de fase aguda y su relación con el cortisol plasmático.

\section{Pacientes y Metodología}

\section{Pacientes}

Durante la epidemia por VRS 2009-2011, seleccionamos lactantes menores de 1 año, previamente sanos sin factores de riesgo, que cursaban con infección de diferente severidad. Todos presentaban fiebre, tos, coriza que había comenzado en las 24 a $72 \mathrm{~h}$ previas, obstrucción bronquial demostrada por la presencia de sibilancias o hiperinflación a la radiografía de tórax y se detectó la presencia de VRS en el aspirado nasofaríngeo (ANF) por inmunofluorescencia indirecta (IFI) o por reacción de polimerasa en cadena (RT-PCR). Los criterios de exclusión fueron lactantes menores de 2 me- ses, co-infección con otros virus o haber recibido corticosteroides inyectable $\mathrm{u}$ oral, $72 \mathrm{~h}$ antes de su ingreso al estudio. El grupo control, seleccionado durante un período no epidémico de infecciones respiratorias, fue un grupo de lactantes sanos que requerían exámenes previos para cirugía menor (hernias inguinales, papilomas pre-auriculares, y/o hemograma control). Pacientes y controles fueron atendidos en el Hospital Roberto del Río y en el centro de salud familiar Agustín Cruz Melo. El estudio fue aprobado por los comités de ética del Hospital Roberto del Río y de la Facultad de Medicina de la Universidad de Chile. Los padres dieron su consentimiento informado para que sus hijos participaran en el estudio.

\section{Aspirado nasofaríngeo}

Con una sonda de alimentación de 8Fr Inttube se aspiró la secreción nasofaríngea y se colocó en $3 \mathrm{ml}$ de solución salina en recipiente refrigerado para su traslado a virología. Una vez agitada y homogeneizada, la muestra se dividió en dos partes iguales, quedando $1,5 \mathrm{ml}$ para estudio virológico. Los $1,5 \mathrm{ml}$ restantes se centrifugaron y se realizó estudio en citómetro de flujo de células totales y conteo diferencial, realizando simultáneamente un extendido celular que se tiñó con May-GrunwaldGiemsa para diferenciar en microscopio óptico los diferentes tipos celulares. El sobrenadante se guardó a $-80^{\circ} \mathrm{C}$ para cuantificar citoquinas.

\section{Estudio viral}

Una vez seleccionados los pacientes con infección respiratoria aguda baja (IRAB) por VRS se obtuvo un segundo ANF para una nueva detección de virus que incluye VRS, Parainfluenza 1,2,3 Influenza A y B, adenovirus y metapneumovirus. En los controles se tomó sólo una muestra de ANF para detección de igual panel viral.

\section{Muestras de sangre}

De 3-5 $\mathrm{ml}$ de sangre heparinizada, se separó 1 $\mathrm{ml}$ de plasma que se guardó a $-80^{\circ} \mathrm{C}$ para cuantificación de citoquinas y de cortisol.

\section{Clasificación de gravedad clínica}

El criterio clínico aplicado para la clasificación de gravedad desde la admisión y durante toda la hospitalización fue modificado de la clasificación previa realizada en el hospital Roberto del Río y publicado por Larrañaga y $\mathrm{col}^{17}$, basado en tres 
parámetros: días de hospitalización, necesidad de oxígeno (días) y la fracción máxima de oxígeno entregada durante la hospitalización. La modificación aplicada en este estudio fue: incluir pacientes ambulatorios y no incluir pacientes de la unidad de cuidados intensivos (UCI).

\section{Cuantificación de citoquinas}

La concentración de citoquinas en el ANF y en plasma fue determinada usando un kit de CBA "Cytokine Beads Assay" (BD Biosciences) de acuerdo al protocolo indicado por el fabricante. Evaluamos las concentraciones de: IL-10, IL-6, TNF- $\alpha$, IL-1 $\beta$, IL-8, IL-12p70, IL-2, IL-4, IL-5, IFN- $\gamma$, e IL-17 mediante citómetro de flujo (FASCan to II con software DIVA).

\section{Cuantificación de cortisol plasmático}

La concentración de cortisol plasmático (n/ $\mathrm{ml}$ ) se determinó por radioinmunoensayo (RIA) (Diagnostic System Laboratories Inc Webster, Texas).

\section{Análisis estadístico}

Los resultados expresados en medianas se analizaron por test no-paramétrico ANOVA KruskalWallis con test de comparación múltiple de Dunn. Las correlaciones fueron analizadas por test de Spearman. Una $\mathrm{p}<0,05$ se consideró significativa.

\section{Resultados}

\section{Pacientes estudiados según gravedad clínica}

Fueron incluidos 49 pacientes con bronquiolitis por VRS, 25 fueron catalogados como graves y 24 como moderados-leves. De estos últimos en 12 la hospitalización no fue necesaria y en la clasificación de gravedad se consideró un puntaje de 1. Al no incluir pacientes de UCI, nuestro puntaje de gravedad máximo fue de 8 .

\section{Características demográficas}

En la Tabla 1 se expresan los resultados obtenidos en número de lactantes estudiados por gravedad, género y edad expresada en mediana (mínimo y máximo). No hubo diferencia significativa entre los tres grupos estudiados.

\section{Recuento total y diferencial de leucocitos en aspirado nasofaríngeo}

La cantidad de leucocitos totales en el ANF en pacientes infectados fue tres veces mayor que en controles $\left(140 / \mathrm{mm}^{3}\right.$ vs $42 / \mathrm{mm}^{3}$ respectivamente). Sin embargo, el porcentaje de granulocitos y de mononucleares fue similar de $80 \%$ y $20 \%$, respectivamente, en todos los grupos (datos no mostrados).

\section{Niveles $(\mathrm{P} / \mathrm{ml})$ de interleuquinas en aspirado nasofaríngeo (ANF)}

La Tabla 2 muestra que IL-6, IL-8 e IL-1- $\beta$ estaban significativamente aumentadas en ambos grupos de pacientes con bronquiolitis por VRS comparadas con el grupo control. En el grupo catalogado como grave el aumento fue significativamente mayor que en el grupo con severidad moderada a leve. En cambio los valores de IL-10 y TNF- $\alpha$ en ANF fueron bajos en todos los grupos y alcanzaron diferencias estadísticamente significativas sólo entre pacientes graves y controles. En todos los lactantes, incluyendo controles sanos, fue posible detectar niveles de IL-6, IL- 8 e IL- $1 \beta$, en cambio IL-10 y TNF- $\alpha$ estaban presentes en un porcentaje mayor de lactantes infectados que en controles (70\% versus 30\%) a pesar de que la cantidad detectada fue muy baja.

\section{Niveles $(\mathrm{P} / \mathrm{ml})$ de interleuquinas en el plasma}

La concentración de IL-6 en el plasma (Tabla 3) fue significativamente mayor en el grupo de pacientes con bronquiolitis grave y moderada comparada con el grupo control. La IL-8 se en-

Tabla 1. Características demográficas de los lactantes

\begin{tabular}{|lcccc|}
\hline & Graves & Moderados-leves & Controles & p \\
\hline $\mathrm{n}$ & 25 & 24 & 25 & NS \\
\hline Género & $15 \hat{\gamma} / 10 q$ & $14 \hat{\jmath} / 10 q$ & $13 \delta^{\lambda} / 12 q$ & NS \\
\hline Edad (meses) & $3(2-10)$ & $3,5(2-9)$ & $3(3-5)$ & NS \\
\hline
\end{tabular}

Edad expresada en medianas (mínima y máxima). 
Tabla 2. Niveles de interleuquinas (P/ml) en el aspirado nasofaríngeo (ANF) de pacientes con bronquiolitis por VRS y controles

\begin{tabular}{|ccccc|}
\hline ANF & $\begin{array}{c}\text { Graves } \\
\mathbf{n = 2 5}\end{array}$ & $\begin{array}{c}\text { Moderados-leves } \\
\mathbf{n = 2 4}\end{array}$ & $\begin{array}{c}\text { Controles } \\
\mathbf{n = 2 5}\end{array}$ & p \\
\hline IL-6 & $326(21-8.271)^{* \dagger}$ & $130(4-3.075)^{*}$ & $11(2-211)$ & $<0,001^{*}$ \\
& & & & $<, 02^{\dagger}$ \\
IL-8 & $8679(245-118.818)^{*+}$ & $2.368(296-55.784)^{*}$ & $596(29-2747)$ & $<0,01^{*}$ \\
& & & & $<, 007^{\dagger}$ \\
IL-1 $\beta$ & $363(8-8.484)^{*+}$ & $157(15-1.470)^{*}$ & $42(4-771)$ & $<0,01^{*}$ \\
& & & & $<0,03^{\dagger}$ \\
IL-10 & $4,0(0-20)^{*}$ & $3,6(2-23)$ & $2,7(0-5)$ & $<0,05^{*}$ \\
\hline TNF- $\alpha$ & $6,4(0-88)^{*}$ & $5,4(0-111)$ & $3,5(0-13)$ & $<0,05^{*}$ \\
\hline
\end{tabular}

Valores están expresados en medianas (mínimo y máximo). * $p$ significativa versus controles ANOVA no paramétrico; ${ }^{\dagger} p$ significativa entre graves versus moderados-leves Mann-Whitney. Límites de detección (P/ml) IL-6=2,5; IL-8=3,6; IL-1- $\beta=7,2$; $\mathrm{IL}-10=3,3 ; \mathrm{TNF}-\alpha=3,7$.

Tabla 3. Niveles de interleuquinas $(\mathrm{P} / \mathrm{ml})$ en plasma de pacientes con bronquiolitis por VRS y controles sanos

\begin{tabular}{|ccccc|}
\hline Plasma & $\begin{array}{c}\text { Graves } \\
\mathbf{n = 2 5}\end{array}$ & $\begin{array}{c}\text { Moderados-leves } \\
\mathbf{n = 2 4}\end{array}$ & $\begin{array}{c}\text { Control } \\
\mathbf{n = 2 5}\end{array}$ & p \\
\hline IL-6 & $14(2-59)^{*}$ & $6(0-60)^{*}$ & $2(0-4)$ & $<0,001$ \\
\hline IL-8 & $10(3-54)^{*}$ & $7(2-36)$ & $4(3-13)$ & $<0,001$ \\
\hline IL-1 $\beta$ & $0(0-3)$ & $1,4(0-6,4)$ & $0(0-4,3)$ & NS \\
IL-10 & $1,7(0-9,1)$ & $1,6(0-8,8)$ & $0(0-4,1)$ & NS \\
\hline TNF- $\alpha$ & $0(0-10,4)$ & $0(0-7,1)$ & $0(0-4,4)$ & NS \\
\hline
\end{tabular}

Valores están expresados en medianas (mínimo y máximo). * $p$ significativa versus controles ANOVA no paramétrico. Límites de detección (P/ml) IL-6=2,5; IL-8=3,6; IL-1- $\beta=7,2 ; \mathrm{IL}-10=3,3 ; \mathrm{TNF}-\alpha=3,7$.

contró aumentada con respecto a grupo control, sólo en pacientes graves. IL-6 e IL-8 se detectaron en prácticamente todos los pacientes, en cambio en los controles la detección fue cercana a la mitad de los lactantes. En sangre no se detectó IL-1 $\beta$, ni TNF- $\alpha$. IL-10 se detectó sólo en un tercio de los enfermos.

Ninguna de la otras interleuquinas estudiadas a saber: IL-12p70, IL-2, IL-4, IL-5, IFN- $\gamma$, e IL-17 presentaron aumento en ANF ni en plasma en pacientes con respecto a grupo control.

Correlación entre la cantidad de interleuquinas cuantificadas en el aspirado nasofaríngeo y en el plasma con la gravedad de la infección

La Figura 1 muestra la correlación entre la gravedad de los pacientes y los niveles de IL- 6 en el ANF de cada uno de ellos $(\mathrm{r}=0,41 ; \mathrm{p}<0,004)$.

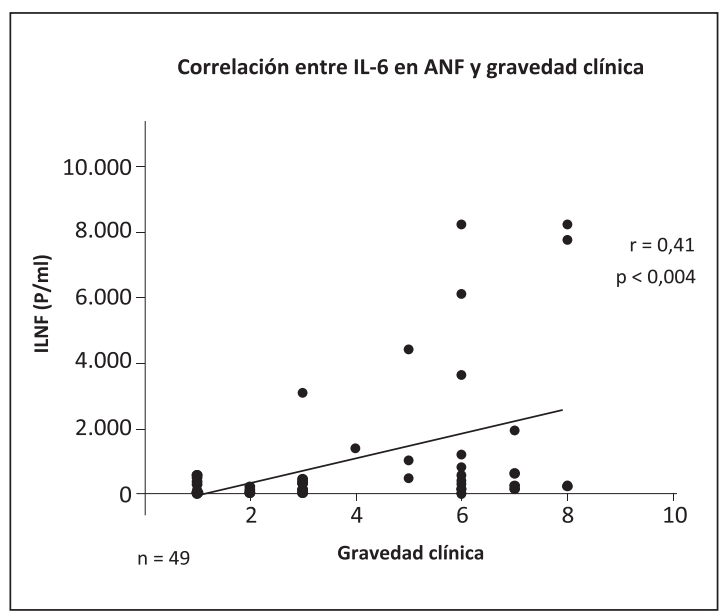

Figura 1. Los 49 pacientes infectados por VRS mostraron correlación positiva $(r=0,41)$ entre la gravedad clínica y la IL-6 en el aspirado nasofaríngeo $(p<0,004)$. 
La Tabla 4 muestra la correlación entre la gravedad de la infección (valores 1-8) con IL-6, IL-8 e IL-1 $\beta$ en el aspirado nasofaríngeo y con IL-6 e IL-8 en el plasma.

El aumento de IL-6 tanto en el plasma como en el ANF fue la que mostró mejor correlación con la gravedad clínica $(\mathrm{r}=0,52 ; \mathrm{p}<0,0001)$.

Las otras ILs presentaron una menor correlación, aunque estadísticamente significativa. No hubo correlación entre la gravedad de la infección con los niveles de TNF- $\alpha$ y de IL-10 encontrados en ANF.

\section{Niveles de cortisol plasmático}

Los lactantes con IRAB grave tenían una mediana de $230 \mathrm{n} / \mathrm{ml}$ significativamente mayor que los lactantes que cursaban con una IRAB moderada-leve de $123(\mathrm{n} / \mathrm{ml}) \mathrm{p}<0,05$ y ambos valores significativamente mayores que la mediana de lactantes sanos con $49(\mathrm{n} / \mathrm{ml}) \mathrm{p}<0,001$ y 0,01 respectivamente (datos no publicados).

\section{Correlación de los niveles de cortisol plasmático con los niveles de interleuquinas.}

La Figura 2 muestra una correlación positiva $(r=0,55)$ entre los niveles plasmáticos de cortisol y de IL-6 ( $<<0,0001)$. No hubo correlación de cortisol plasmático con los niveles plasmáticos de IL-8. Las interleuquinas cuantificadas en el ANF no mostraron correlación con el nivel de cortisol plasmático de los pacientes.

\section{Discusión}

En este estudio analizamos la respuesta mediada por interleuquinas de respuesta inmune innata (IL-6, IL-8, IL-1- $\beta$, TNF- $\alpha$, IL-12, e IL-10) y de respuesta inmune adaptativa de tipoTh-1(IFN- $\gamma$, IL-2) y tipo Th-2 (IL-4, IL-5, IL-10) de lactantes menores de un año infectados por primera vez con VRS, de características demográficas similares pero con diversa evolución clínica. Las interleuquinas de fase aguda de respuesta inmune innata IL-6, IL-8 estaban aumentadas en el ANF y plasma y sólo IL-1 $\beta$ en ANF (Tablas 2 y 3 ) en los pacientes con VRS comparados con el grupo control. Las muestras de ANF y de plasma para cuantificar las interleuquinas fueron tomadas en los primeros días de enfermedad (2-4 días) y la clasificación de la gravedad de la enfermedad fue obtenida cuando
Tabla 4. Correlación entre la gravedad de la infección con IL-6, IL-8 e IL-1 $\beta$ en el aspirado nasofaríngeo y con IL-6 e IL-8 en el plasma

\begin{tabular}{|ccc|}
\hline Interleuquina & Spearman $\mathbf{r}$ & Significancia \\
\hline IL-6 ANF & 0,41 & $\mathrm{p}<0,004$ \\
IL-8ANF & 0,33 & $\mathrm{p}<0,02$ \\
IL-1 $\beta$ ANF & 0,30 & $\mathrm{p}<0,04$ \\
IL-6 plasma & 0,52 & $\mathrm{p}<0,0001$ \\
\hline IL-8 plasma & 0,34 & $\mathrm{p}<0,02$ \\
\hline
\end{tabular}

Test de rango de correlación de Spearman (r).

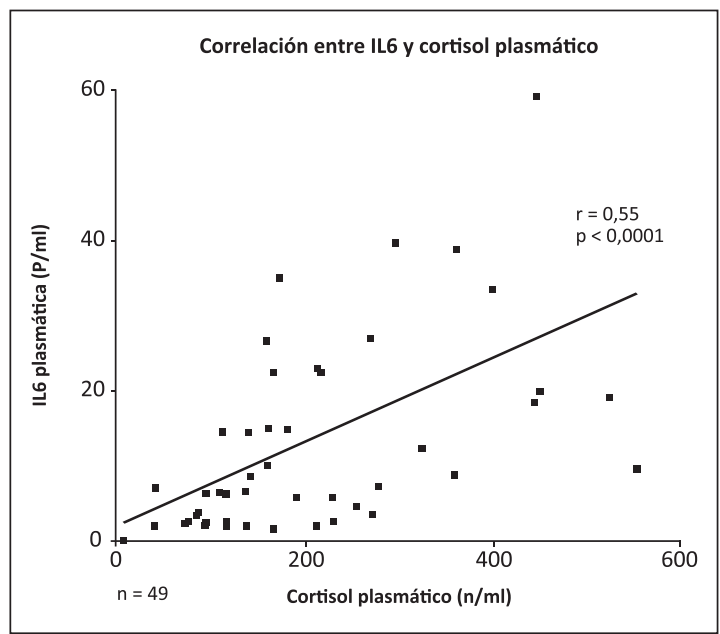

Figura 2. Los 49 pacientes infectados por VRS mostraron correlación positiva $(r=0,55)$ entre cortisol plasmático y nivel de IL-6 en el plasma $(p<0,0001)$.

el paciente fue dado de alta. La IL-6 plasmática y en ANF fue la que mostró mejor correlación con la gravedad del paciente. Este resultado es coincidente con lo observado en otros estudios ${ }^{7,8}$ donde se comparó lactantes graves sometidos a ventilación mecánica con lactantes no graves. La IL-6 es una citoquina con múltiples funciones, producida por células linfoides y no linfoides. En la vía aérea puede ser producida por células epiteliales y mononucleares especialmente monocitosmacrófagos. En nuestro estudio encontramos que la cantidad producida localmente (ANF) fue mayor en relación al plasma incluso en lactantes controles (5:1). En los lactantes infectados esta relación aumenta aún más $(20: 1)$. 
IL-8 es una citoquina con actividad quimiotáctica para neutrófilos, su aumento en el ANF explica el aumento de estas células que encontramos en nuestros pacientes, resultados concordantes con otros descritos ${ }^{24}$. Sin embargo, su relación con la gravedad de la infección es controversial. Smyth y col encontraron una asociación entre la cantidad de IL-8 producida localmente en las vías aéreas y cuantificadas por RNAm y la gravedad de la infección por VRS ${ }^{25}$. En cambio, Bennett y col encontraron un aumento significativo en el aspirado nasofaríngeo de ILs proinflamatorias pero que no tenía relación con la gravedad de la enfermedad ni con la presencia del VRS ${ }^{26}$. La presencia de neutrófilos sí está relacionada con la inflamación de la vía aérea en la bronquiolitis por VRS ${ }^{27-28}$. Nuestro estudio mostró un aumento en la cantidad de neutrófilos en la vía aérea, que podría estar relacionada con la IL-8, ya que la IL17 no estaba aumentada en el ANF a diferencia de lo descrito en lactantes muy graves sometidos a ventilación mecánica ${ }^{29}$.

La producción de IL-1 $\beta$, IL-10 y de TNF- $\alpha$ fue encontrada en el ANF de los pacientes, presentando sólo IL- $1 \beta$ cantidades significativamente altas en pacientes más graves. La ausencia de IL-1 $\beta$ y de TNF- $\alpha$ en el plasma, se podría explicar porque ninguno de estos pacientes hizo una infección grave necesaria de ser tratada en unidad de cuidados intensivos, ya que ambas citoquinas se asocian a cuadros clínicos extremadamente severos en su evolución ${ }^{30,31}$.

La cuantificación de interleuquinas es una técnica fácil de realizar (citometría de flujo o ELISA) sin embargo, la gran variabilidad individual en su producción y su corto tiempo de acción, dificulta su aplicabilidad clínica.

Es importante destacar que investigaciones previas $^{21-23}$ han demostrado que la producción especialmente de IL-6, y también IL-8, IL-1 $\beta$ y TNF- $\alpha$ en infecciones virales, estrés y ejercicio activan el eje hipotálamo-hipófisis-suprarrenal, liberando cortisol que tiene acción antiinflamatoria. La respuesta inflamatoria "controlada" es esencial como mecanismo para eliminar el agente infeccioso, cuando esta respuesta es exagerada se produce daño tisular. En este estudio encontramos una correlación positiva del cortisol plasmático con la gravedad de la infección y con el nivel de IL-6 plasmática, mostrado en Figura 2. La falta de acción antiinflamatoria del cortisol en la bronquiolitis por VRS ha sido demostrada en múltiples estudios clínicos sobre tratamiento de la IRAB por VRS con cortico esteroides ${ }^{32-34}$. Recientemente, Hinsey y $\mathrm{col}^{35}$ publicaron que en células de cultivo había una inhibición directa del VRS en la acción antiinflamatoria de los corticoides. En los lactantes estudiados por nosotros, encontramos, en relación a la gravedad del paciente, un aumento del receptor de glucocorticoides beta (GR- $\beta$ ) que es inactivo en la respuesta a corticoides ${ }^{36}$. Esto podría explicar en parte, que el cortisol no tenga una acción antiinflamatoria en un subgrupo de pacientes que presenta una evolución de gravedad. Por otro lado, en pacientes adultos con infección por VRS que fueron tratados con esteroides orales por enfermedad basal, no hubo diferencia en la cantidad de IL-6 e IL-8 en el suero y en el ANF y tampoco en la evolución clínica con pacientes no tratados con esteroides ${ }^{37}$, confirmando así que el corticoide no tiene acción anti-inflamatoria frente al VRS.

En conclusión, los lactantes infectados con VRS presentan desde el comienzo de su enfermedad un aumento de interleuquinas pro-inflamatorias en el ANF y en el plasma que es significativamente mayor en lactantes que evolucionan con mayor gravedad. Así, cuantificar las interleuquinas en los primeros días de la enfermedad podría servir como marcadores de pronóstico. El aumento de IL-6 podría explicar en parte el aumento de cortisol plasmático y su relación con la gravedad del cuadro clínico.

\section{Referencias}

1. Avendaño LF, Palomino MA, Larrañaga C. Surveillance for respiratory syncytial virus in infants hospitalized for acute lower respiratory infection in Chile. J Clin Microbiol 2003; 41: 4879-82.

2. Falsey AR, Hennessey PA, Formica MA, Cox C, Walsh EE. Respiratory Syncytial Virus infection in elderly and high risk adults. N Engl J Med 2005; 352: 1749-59.

3. Hoffman SJ, Laham FR, Polack FP. Mechanism of illness during respiratory syncytial virus infection: the lungs, the virus and the immune response. Microbes and Infection 2004; 6: 762-7.

4. Duncan CB, Walsh EE, Peterson DR, Lee FE-H, Falsey AR. Risk factors for respiratory failure associated with respiratory syncytial virus infection in adults J Infect Dis 2009; 200: 1242-46. 
5. Collins PL, Melero JA. Progress in understanding and controlling respiratory syncytial virus: Still crazy after all these years Virus Research 2011; 162: 80-99.

6. Sheeran P, Jafri H, Carubelli C, Saavedra J, Krisher K, Sánchez PJ, Ramilo O. Elevated cytokine concentrations in the nasopharyngheal and tracheal secretions of children with respiratory syncytial virus disease. Pediatr Infect Dis J 1999; 18: 115-22.

7. McNamara PS, Flanagan BF, Selby AM, Hart CA, Smyth RL. Pro and anti-inflammatory responses in respiratory syncytial virus bronquiolitis. Eur Respir J 2004; 23: 10612.

8. Faber TE, Groen H, Welfing M, Jansen KJG, Bont LJ. Specific increase in local IL-17 production during recovery from primary RSV bronchiolitis. J Med Virol 2012; 84: $1084-8$.

9. Openshaw PJM, Tregoning JS. Immune responses and disease enhancement during respiratory syncytial virus infection. Clin Microb Rev 2005; 18: 541-55.

10. Arnold R, König B, Gallati H, Werchau H, König W. Interleukin-8, Interleukin-6, and soluble tumor necrosis factor receptor type release from a human pulmonary epithelial cell line (A549) exposed to respiratory syncytial virus. Immunology 1995; 82: 364-72.

11. Fonceca AM, Flanagan BF, Trinick R, Smyth RL, McNamara PS. Primary airway epithelial cultures from children are highly permissive to respiratory syncytial virus infection Thorax 2012; 67: 42-8.

12. Díaz P, Calhoun WJ, Hinton K, Avendaño LF, Gaggero A, Simon V, et al. Differential effects of respiratory syncytial virus and adenovirus on mononuclear cell cytokine responses. Am J Respir Crit Care Med 1999; 160: 1157-64.

13. Roman M, Calhoun WJ, Hinton KM, Avendaño LF, Simon V, Escobar AM, et al. Respiratory syncytial virus infection in infants is associated with predominant Th-2 like response. Am J Respir Crit Care Med 1997; 156; 160: 1263-8.

14. Aberle JH, Aberle SW, Rebhandl W, Pracher E, Kundi M, Popow-Kraupp T. Decreased interferon-gamma response in respiratory syncytial virus compared to other respiratory viral infections in infants. Clin Exp Immunol 2004; 137: 146-50.

15. Bont L, Heijnen CJ, Kavelaars A, Van Aalderen WMC, Brus M, Draaisma JT, et al. Peripheral blood cytokine responses and disease severity in respiratory virus bronchiolitis Eur Respir J 1999; 14: 144-9.

16. Leggs JP, Hussain IR, Warner JA, Johnston SL, Warner JO. Type 1 and Type - 2 cytokine imbalance in acute respiratory syncytial virus bronchiolitis. Am J Respir Crit Care Med 2003; 168: 633-9.

17. Pinto R, Arredondo SM, Bono MR, Gaggero AA, Díaz
PV. T helper 1/Thelper 2 cytokine imbalance in respiratory syncytial virus infection is associated with increase endogenous plasma cortisol Pediatrics 2006; 117e 87886.

18. Larrañaga CL, Ampuero SL, Luchsinger VF, Carrión FA, Aguilar NV, Morales PR, et al. Impaired immune response in severe human lower tract respiratory infection by respiratory syncytial virus Ped Infect Dis J 2009 28; 867-73.

19. Welliver TP, Garofalo RP, Hosakote Y, Hintz KH, Avendaño LF, Sanchez K, et al. Severe human lower respiratory tract illness caused by respiratory syncytial virus and influenza virus is characterized by the absence of pulmonary cytotoxic lymphocyte responses. J Infect Dis 2007; 195: 1126-36.

20. Haddad JJ, Saadé NE, Safieh-Garabedian B. Cytokines and neuro-immune-endocrine interactions: a role for the hypoyhalamic-pituitary-adrenal revolving axis. J Neuroimmunol 2002; 133: 1-19.

21. Bethin KE, Vogt SK, Muglia LJ. Interleukin-6 is an essential, corticotrophin releasing hormone-independent, stimulator of the adrenal axis during immune system activation. PNAS 2000; 16: 9317-22.

22. Silverman MN, Miller AH, Biron CA, Pearce BD. Characterization of an Interleukin-6 and Adrenocorticotropin-dependent, immune-to- adrenal pathway during viral infection. Endocrinology 2004; 145: 3580-9.

23. Steensberg A, Fisher CP, Keller C, Moller K, Pedersen BK. IL-6 enhances plasma IL-1ra, IL-10 and cortisol in humans. Am J Physiol Endocrinol Metab 2003; 285: E433-E7.

24. Smyth RL, Mobbs KJ, O’Hea U, Ashby D, Hart CA. Respiratory syncytial virus bronchiolitis: disease severity. Interleukin-8 and virus genotype. Ped Pulm 2002; 33: 339-46.

25. McNamara PS, Ritson P, Selby A, Hart CA, Smyth RL. Broncoalveolar lavage cellularity in infants with severe respiratory syncytial virus bronchiolitis. Arch Dis Child 2003; 88: 922-6.

26. Bennett BL, Garofalo RP, Cron SG, Hosakote YM, Atmar $\mathrm{RL}$, Macias CG, et al. Immunpathogenesis of respiratory syncytial virus bronchiolitis. J Infect Dis 2007; 195: 1532-40.

27. Emboriadou M, Hatzistilianou M, Magnisali Ch, Sakelaropoulou A, Exintari M, Conti P, et al. Human neutrophil elastase in RSV bronchiolitis. Ann Clin Lab Sci 2007; 37: 79-84.

28. Yasui K, Baba A, Iwasaki Y, Kubo T, Aoyama K, Mori T, et al. Neutrophil-mediated inflammation in respiratory syncytial viral bronchiolitis. Ped Int 2005; 47: 190-5.

29. Mukherjee S, Lindell DM, Berlin AA, Morris SB, Shanley 
TP, Hertshenson MB, et al. IL-17 induced pulmonary pathogenesis during respiratory viral infection and exacerbation of allergic disease. Am J Pathol 2011; 179: 248-58.

30. Midulla F, Villani A, Panuska JR, Dab I, Kolls JK, Merolla $\mathrm{R}$, et al. Respiratory syncytial virus lung infection in infants: immunoregulatory role of infected alveolar macrophages. J Infect Dis 1993; 168: 1515-9.

31. Puthothu B, Bierbaum S, Kopp MV, Forster J, Heinze J, Weckmann M, et al. Association of TNF- $\alpha$ with severe respiratory syncytial virus infection and bronchial asthma. Pediatr Allergy Immunol 2009; 20: 157-63.

32. Emers MJ, Rovers MM, van Woensel JB, Kimpen JL, Bont LJ, RSV corticosteroid study group. The effect of high dose inhaled corticosteroids on wheeze in infants after respiratory syncytial virus infection: randomized double blind placebo controlled trial. BMJ 2009; 338: b897.
33. Patel H, Platt R, Lozano JM, Wang EE. Glucocorticoids for acute viral bronchiolitis in infants and young children. Cochrane Database Syst Rev 2004; 3: CD004878.

34. Bonville CA, Mehta PA, Krilov LR, Rosenberg HF, Domachowske JB. Epithelial cells infected with respiratory syncytial virus are resistant to the anti-inflammatory effect of hydrocortisone Cel Immunol 2001; 213: 134-40.

35. Hinsey A, Alexander J, Corry J, Adams KM, Clagget AM, Traylor ZP, et al. Respiratory syncytial virus represses glucocorticoid receptor-mediated gene activation. Endocrinology 2011; 152: 483-94.

36. Díaz PV, Pinto RA, Mamani R, Uasapud P, Bono MR, Gaggero A, et al. Increased expression of the glucocorticoid receptor $\beta$ in infants with RSV bronchiolitis. Pediatrics 2012; 130; e804-11.

37. Lee eF-H, Walsh EE, Falsey AR. The effect of steroid use in hospitalized adults with respiratory syncytial virusrelated illness. Chest 2011; 140: 1155-61. 